\title{
Ingestão do cálcio na obesidade de mulheres atendidas pelo Sistema Único de Saúde ${ }^{1}$
}

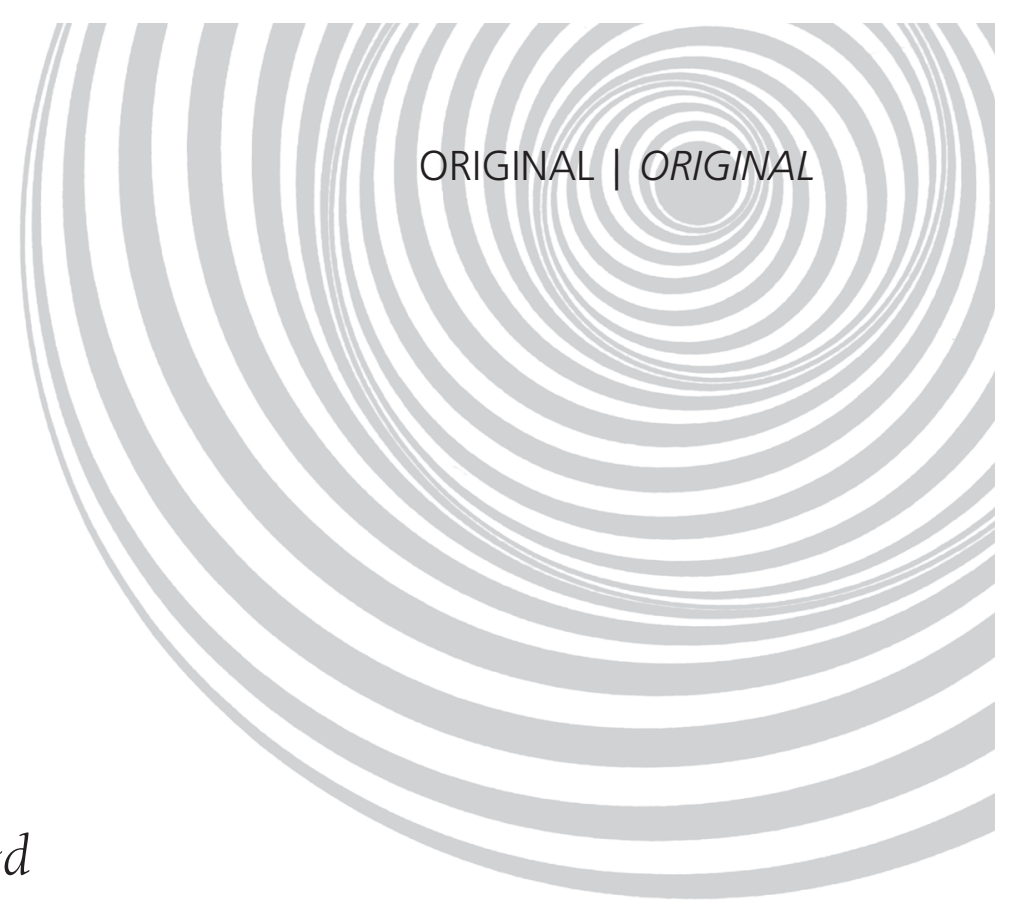

\author{
Calcium intake in obese women treated \\ at Sistema Único de Saúde, Brazil
}

Patricia Maria Candido SILVA ${ }^{2}$

Cyro Rego CABRAL JUNIOR ${ }^{3}$

Sandra Mary Lima VASCONCELOS²

RES U M O

\section{Objetivos}

Verificar a associação entre consumo de cálcio e obesidade em uma amostra de mulheres obesas ou eutróficas.

\section{Métodos}

Por meio de inquéritos alimentares, estimou-se a ingestão de cálcio de 177 mulheres hipertensas e/ou diabéticas mediante recordatórios de $24 \mathrm{~h}$ e frequência de consumo alimentar. Estudo caso-controle teve o grupo-estudo composto por 102 mulheres obesas e o grupo-controle por 75 mulheres eutróficas. Os dados de ingestão foram comparados às recomendações dietéticas e às porções de alimentos de origem animal, recomendados pela pirâmide alimentar brasileira, que são fontes de cálcio. Na análise estatística dos dois grupos segundo faixa etária, utilizou-se o coeficiente de correlação linear simples de Pearson com nível de significância de até $5 \%$ de probabilidade de erro experimental.

\section{Resultados}

A ingestão quantitativa de cálcio foi semelhante entre os grupos e igualmente insuficiente para atingir as recomendações nutricionais. A análise da frequência de consumo de fontes de cálcio revelou que os dois grupos apresentavam consumo inferior a três porções/dia, merecendo destaque o grande número que referiu "raramente" consumir tais alimentos.

\section{Conclusão}

Não foi observada nessa população a associação entre obesidade e ingestão de cálcio. Seu consumo foi insuficiente para atingir as quantidades recomendadas, um achado relevante. Considerando a idade das

1 Trabalho desenvolvido em projeto de pesquisa para o SUS (PPSUS/CNPq/FAPEAL), processo 007/2004-PPSUS-AL.

${ }^{2}$ Universidade Federal de Alagoas, Faculdade de Nutrição, Laboratório de Nutrição em Cardiologia. Campus A.C. Simões, BR 104 Norte, km 97, Tabuleiro dos Martins, 57072-970, Maceió, AL, Brasil. Correspondência para/Correspondence to: S.M.L. VASCONCELOS.E-mails: <sandramary@uol.com.br>; <sandra-mary@hotmail.com>.

${ }^{3}$ Universidade Federal de Alagoas, Faculdade de Nutrição. Maceió, AL, Brasil. 
mulheres estudadas e o diagnóstico de hipertensão e diabetes, é evidente a necessidade de atividades de educação nutricional para estimular o consumo de fontes alimentares de cálcio com o intuito de auxiliar no controle da hipertensão e de prevenir agravos importantes, como a obesidade e a osteoporose.

Termos de indexação: Cálcio. Ingestão dietética. Obesidade.

\section{A B S T R A C T}

\section{Objective}

The objective of this study was to assess the relationship between calcium intake and obesity in a sample of obese and normal weight women.

\section{Methods}

The 24-hour recall and food frequency questionnaire were used to determine the calcium intake of 177 hypertensive and/or diabetic women. This was a case-control study. The study group consisted of 102 obese women and 75 normal weight women (control). Intake data were compared with the dietary reference intakes and with the animal-source food portions, which are the sources of calcium recommended by the Brazilian food pyramid. The Pearson correlation coefficient with a significance level of $5 \%$ of experimental error probability was used for the statistical analysis of both groups according to age groups.

\section{Results}

Calcium intake was similar between the groups and both failed to reach the recommended intake levels. Analysis of the intake frequency of calcium-rich foods showed that both groups consumed less than 3 portions a day. The high percentage of women who reported hardly ever eating such foods is worthy of notice.

\section{Conclusion}

An association between obesity and calcium intake was not found in this population. Calcium intake failed to reach the recommended levels, which was a relevant finding. Considering the age of the studied women and the diagnoses of diabetes and hypertension, there is a clear need for nutrition education activities that encourage the intake of calcium-rich foods, which aid in the control of hypertension, as well as in the prevention of important diseases, such as obesity and osteoporosis.

Indexing term: Calcium. Dietary intake. Obesity.

\section{N T R O D U ÇÃ O}

A obesidade constitui um grave problema de saúde pública no mundo, e tem sido associada ao risco de hipertensão arterial, doenças cardiovasculares, diabetes tipo 2, doenças da vesícula biliar, alguns tipos de câncer, osteoartrite, apneia do sono e outras desordens ${ }^{1-4}$. Trata-se de uma enfermidade crônica multifatorial caracterizada por fenômenos sociais, ambientais, fisiológicos e clínicos de diversas origens que induzem ao desequilíbrio crônico entre a energia ingerida e o gasto energético. Nesse desequilíbrio podem estar implicados diversos fatores relacionados ao estilo de vida, alterações neuroendócrinas e uma predisposição genética sobre a qual atuam fatores ambientais $^{5}$ que, na população brasileira, levaram a uma transição nutricional devido ao estilo de vida, caracterizado pela diminuição nos níveis de atividade física e pela ingestão de alimentos de alta densidade energética e baixo conteúdo de nutrientes ${ }^{6,7}$.

Na relação nutrição-obesidade, destaca-se o perfil de consumo alimentar que, com a dieta afluente $^{8}$, incluiu o decréscimo do consumo de alimentos de origem vegetal e o aumento daqueles de origem animal (e, portanto, de proteína e gordura saturada), além do aumento de alimentos processados e fontes de carboidratos simples, gorduras e sal. A ingestão de energia e de nutrientes específicos tem despontado como importantes contribuintes nessa relação de causalidade ${ }^{1,7}$. Além da influência dos macronutrientes, estudos nessa área relatam a presença de distúrbios na ação de minerais envolvidos no metabolismo 
energético que contribuem para a fisiopatologia da obesidade, destacando-se o zinco, o magnésio, o potássio e, principalmente, o cálcio9 ${ }^{9}$.

A participação do cálcio na fisiopatologia da obesidade e seu elo com a osteoporose foi discutida numa revisão publicada no ano de 2009, na qual Rosen \& Klibanski ${ }^{10}$ discutem que o hipotálamo modula a reserva de gordura e de osso por meio do Sistema Nervoso Simpático (SNS) através da regulação do apetite, sensibilidade à insulina, e remodelação do esqueleto, e que a medula óssea, osteoblastos e adipócitos originam-se do mesmo precursor, de modo que a obesidade e a osteoporose não só podem coexistir, mas que o excesso de gordura visceral pode contribuir para a perda da massa óssea. Apontam estudos que corroboram essa ligação, uma vez que encontraram maior risco de fraturas em portadores de síndrome metabólica e importante redução da massa óssea em indivíduos que se submeteram à cirurgia bariátrica, indicando a necessidade de avaliar a massa óssea na monitorização de indivíduos obesos.

A associação do cálcio à doença da obesidade tem motivado muitos estudos observacionais e de intervenção. Dados epidemiológicos sugerem que indivíduos com alta ingestão de cálcio têm menor prevalência de sobrepeso, obesidade e resistência à insulina ${ }^{11}$. Os estudos nessa linha se iniciaram com McCarron ${ }^{12}$, que verificou, com base nos dados do National Health and Nutrition Examination Survey (NHANES I), uma associação inversa entre ingestão de cálcio e peso corporal na população norte-americana. Zemel et al. ${ }^{13}$, avaliando dados do NHANES III, observaram que, após ajuste pela idade, gênero, raça e consumo de energia, o risco de obesidade era reduzido em $85 \%$ entre aqueles indivíduos que tinham ingestão adequada de cálcio.

Recentemente Dubnov-Raz \& Berry ${ }^{14}$, revisando o tratamento da obesidade, citam 24 estudos publicados de 2000 a 2007 cujos resultados são conflitantes: associação positiva, negativa ou nenhuma associação da ingestão de cálcio com perda ou ganho de peso corporal. Da mesma forma Major et al. ${ }^{15}$ publicaram um documento com as discussões de pesquisadores durante simpósio sobre cálcio e obesidade; analisando as conclusões de 19 estudos sobre o tema, encontraram que elas também divergiam entre positiva, negativa e nenhuma associação.

Existem dois mecanismos fisiológicos propostos para explicar como a ingestão de cálcio influi no peso e na quantidade de gordura corporal: (1) a diminuição da absorção de cálcio, consequente à menor ingestão dietética, induz ao aumento do Paratormônio (PTH) e da 1,25-di-hidroxi vitamina $\mathrm{D}_{3}$ ou calcitriol, que aumentam o influxo do cálcio intracelular no tecido adiposo, estimulando a expressão da enzima ácido graxo sintetase, e assim inibindo a lipólise, promovendo a lipogênese e diminuindo a oxidação de gorduras; e (2) a complexação do cálcio com ácido graxo no intestino, formando sabões insolúveis excretados pelas fezes e portanto diminuindo a absorção de gordura ${ }^{11,16-18}$. O primeiro mecanismo tem sido apontado como o mais consistente para explicar como a baixa ingestão dietética de cálcio contribuiria para o ganho de peso.

Nessa perspectiva, este estudo teve como objetivo avaliar e comparar a ingestão de cálcio entre mulheres obesas e eutróficas para verificar a possível associação entre ingestão de cálcio e peso corporal.

\section{M É TOD O S}

A amostra foi obtida do banco de dados dos pacientes estudados no projeto de pesquisa para o Sistema Único de Saúde (SUS) Hábitos Alimentares e Estado Nutricional de Hipertensos e Diabéticos. Uma Contribuição às Ações de Controle de Fatores de Risco Cardiovascular do HIPERDIA/MS do Município de Flexeiras/AL (PPSUS/CNPq/FAPEAL/SESAU-AL), aprovado pelo comitê de ética em pesquisa da UFAL. Nessa pesquisa foram estudados 476 pacientes portadores de Hipertensão Arterial Sistêmica (HAS) e/ou diabetes mellitus, dos quais foram obtidos Inquéritos Dietéticos tipo Recordatório de 24h (IDR24h), 
com auxílio de registro fotográfico ${ }^{19}$; os dados foram analisados no software Virtual Nutri ${ }^{\circledR 20}$, no qual se obtiveram os níveis de ingestão de energia e nutrientes, inclusive de cálcio.

Com base nesse banco de dados foram selecionados os pacientes do gênero feminino, com IDR24h analisados e com Índice de Massa Corporal (IMC) na faixa de eutrofia ou de obesidade segundo critérios do Sistema de Vigilância Alimentar e Nutricional do Ministério da Saúde (SISVAN-MS) ${ }^{21}$, que classifica segundo faixa etária: adulto ( $\geq 20$ e $<60$ anos): $I M C \geq 18,5 \mathrm{~kg} / \mathrm{m}^{2}$ e $<25 \mathrm{~kg} / \mathrm{m}^{2}$ (eutrofia) e IMC $\geq 30 \mathrm{~kg} / \mathrm{m}^{2}$ (obesidade); e idoso ( $\geq 60$ anos): IMC $>22 \mathrm{~kg} / \mathrm{m}^{2}$ e $<27 \mathrm{~kg} / \mathrm{m}^{2}$ (eutrofia) e IMC $\geq 27 \mathrm{Kg} / \mathrm{m}^{2}$ (sobrepeso).

Foram excluídos os pacientes do sexo masculino e as mulheres com IMC em faixas de sobrepeso e adulto IMC de 25 a $30 \mathrm{~kg} / \mathrm{m}^{2}$ ) e baixo peso (adulto $\mathrm{IMC}<18,5 \mathrm{~kg} / \mathrm{m}^{2}$ e idoso IMC $\leq 22$ $\mathrm{kg} / \mathrm{m}^{2}$ ), que contabilizaram 299 pacientes excluídos. Para atender ao propósito deste estudo, chegou-se a um total de 102 mulheres obesas para o Grupo-Estudo (GE) e 75 mulheres eutróficas para o Grupo-Controle (GC), totalizando 177 mulheres. A amostra distribuiu-se em 154 hipertensas (87\%), 13 diabéticas e hipertensas (7\%) e 10 diabéticas (6\%). Essas pacientes eram acompanhadas pelas equipes de saúde da família através do HIPERDIA, estavam clinicamente controladas e monitorizadas, com ou sem medicação, dependendo da condição clínica. Quando necessário, as pacientes recebiam a medicação disponibilizada para o HIPERDIA, que constitui de um anti-hipertensivos hidroclorotiazida $25 \mathrm{mg}$, propanolol 40mg e captopril 25mg; e (2) hipoglicemiantes orais glibenclamida $5 \mathrm{mg}$ e metformina $850 \mathrm{mg}$, em posologia individualizada e a critério médico.

Das 177 mulheres selecionadas, dispunha-se de um banco de dados com 233 IDR24H - um IDR24H de $100 \%$ dessa amostra (177) e mais 46 com um $2^{\circ}$ IDR24H, de modo que $25 \%$ da amostra tinham 2 IDR24H - para avaliação da frequência de ingestão inadequada de cálcio, pelo método da Estimated Adequate Intake (EAR) como ponto de corte. Esse método requer a repetição do IDR24H em pelo menos $20 \%$ da amostra para avaliação da ingestão de nutrientes de um grupo de indivíduos, uma vez que é necessário calcular a variância intrapessoal e interpessoal e o fator de ajuste para que as médias de consumo sejam comparadas às Dietary Reference Intakes (DRI) (ingestão dietética de referência), no caso do cálcio à Ingestão Adequada (Al)22.

Além dos IDR24H, os grupos foram avaliados quanto à ingestão de fontes alimentares de cálcio de alta biodisponibilidade; para isso foi aplicado inquérito dietético qualitativo de frequência de consumo alimentar de leite e derivados (FCA-LD) em 20\% de cada grupo estudado por meio de sorteio, o que correspondeu a 20 mulheres do GE e 15 mulheres do GC.

A ingestão de cálcio pelos grupos, nos dois tipos de inquéritos dietéticos, foi avaliada pelas recomendações de ingestão adequada estabelecidas pelas $\left.D R\right|^{23}$, segundo faixa etária (19-50 anos $\mathrm{Al}=1000 \mathrm{mg} \mathrm{e}>51$ anos $\mathrm{Al}=1200 \mathrm{mg}$ por dia), o que implicou subdividir obesas (Ob) e eutróficas (Eu) nos seguintes grupos: Ob1 = obesas, 19-50 anos; Ob2 = obesas >51 anos; Eu1 = eutróficas, $19-50$ anos; Eu2 $=$ eutróficas $>51$ anos.

Para análise dos dados obtidos foi utilizado o programa estatístico SPSS 13.0. Além das estatísticas descritivas, os grupos de pacientes eutróficas e obesas foram analisados pelo coeficiente de correlação linear simples de Pearson segundo faixa etária. Adotou-se como nível de significância até $5 \%$ de probabilidade de erro experimental.

O projeto foi aprovado pelo comitê de ética da Universidade Federal de Alagoas, processo no 013287/2004-11, e os pacientes participaram da pesquisa mediante Termo de Consentimento Livre e Esclarecido.

\section{RES U LTA DOS}

Dentre as 177 pacientes do gênero feminino estudadas, as 102 obesas distribuíram-se em 31 no grupo Ob1 e 71 no grupo Ob2, e as 75 
eutróficas distribuíram-se em 21 no grupo Eu1 e 54 no grupo Eu2 (Tabela 1).

Os testes de correlação entre as variáveis antropométricas e entre estas e a ingestão de cálcio por faixa etária, comparando as obesas com as eutróficas (Tabela 2), revelaram uma associação positiva entre peso e altura $(p=0,04)$ e entre peso e IMC $(p=0,00)$ na faixa etária de 19 a 50 anos. Entre as mulheres com idade $\geq 51$ anos, verificou-se uma associação negativa entre idade e peso $(p=0,02)$, idade e altura $(p=0,04)$ e idade e IMC $(p=0,038)$; e uma associação positiva entre peso e altura $(p=0,00)$, peso e IMC $(p=0,00)$ e entre altura e ingestão de cálcio $(p=0,03)$. Considerando que, na segunda faixa etária, a altura associou-se diretamente à ingestão de cálcio e ao aumento do peso, que por sua vez associou-se positivamente ao IMC, pode-se inferir uma tendência de maior ingestão de cálcio à medida que aumenta o IMC. No entanto, a ingestão desse mineral foi deficiente (abaixo do recomendado), como será apresentado a seguir. A ingestão de cálcio entre as participantes do Ob2 apresentou correlação negativa com a idade e IMC, e positiva com peso e altura, sendo com esta última significante, como supracitado; entre as do Ob1, a ingestão de cálcio correlacionou-se negativamente com peso e IMC e positivamente com a altura, embora não significativamente (Tabela 2).

Uma vez subdivididos os grupos $\mathrm{Ob}$ e Eu com base na faixa etária de recomendação de cálcio, verificou-se que a avaliação da ingestão desse nutriente em cada um dos subgrupos foi realizada cobrindo o mínimo de $20 \%$ de um segundo IDR24H estabelecido para o método da "EAR como ponto de corte para grupos", exceto no grupo Eu1, em que um paciente não completou o protocolo, como segue: Ob1= 7 ( $22,5 \%$ de 31$)$;

Tabela 1. Distribuição dos grupos estudados segundo idade e dados antropométricos. Flexeiras (AL), 2007.

\begin{tabular}{|c|c|c|c|c|c|c|c|c|}
\hline \multirow{3}{*}{ Variáveis } & \multicolumn{4}{|c|}{ Obesas $(n=102)$} & \multicolumn{4}{|c|}{ Eutróficas $(n=75)$} \\
\hline & \multicolumn{2}{|c|}{ Ob1: $19-50$ anos $(n=31)$} & \multicolumn{2}{|c|}{ Ob2: $\geq 51$ anos $(n=71)$} & \multicolumn{2}{|c|}{ Eu1: $19-50$ anos $(n=21)$} & \multicolumn{2}{|c|}{ Eu2: $\geq 51$ anos $(n=54)$} \\
\hline & $\mathrm{M}$ & DP & $\mathrm{M}$ & DP & $\mathrm{M}$ & DP & $\mathrm{M}$ & DP \\
\hline Idade (anos) & 41,54 & 6,69 & 63,65 & 7,00 & 40,52 & 7,63 & 66,41 & 10,63 \\
\hline Peso (kg) & 84,26 & 10,83 & 73,87 & 7,65 & 52,89 & 5,81 & 51,59 & 6,58 \\
\hline Altura (m) & 1,56 & 0,06 & 1,49 & 0,05 & 1,52 & 0,07 & 1,49 & 0,08 \\
\hline IMC $\left(\mathrm{kg} / \mathrm{m}^{2}\right)$ & 36,17 & 4,20 & 33,43 & 2,47 & 22,69 & 1,25 & 23,02 & 1,14 \\
\hline
\end{tabular}

M: média; DP: desvio-padrão; IMC: índice de massa corporal; Ob obesas; EU: eutróficas.

Tabela 2. Análise de correlação entre variáveis estudadas nas mulheres obesas versus eutróficas segundo faixa etária. Flexeiras (AL), 2007.

\begin{tabular}{|c|c|c|c|c|c|c|c|c|c|}
\hline \multirow{3}{*}{ Variáveis } & & \multicolumn{8}{|c|}{ Mulheres obesas versus eutróficas } \\
\hline & & \multicolumn{4}{|c|}{$\begin{array}{c}\text { Mulheres } 19 \text { a } 50 \text { anos } n=52 \\
\text { ( } 31 \text { obesas vs } 21 \text { eutróficas) }\end{array}$} & \multicolumn{4}{|c|}{$\begin{array}{l}\text { Mulheres } \geq 51 \text { anos } n=125 \\
\text { (71 obesas vs } 54 \text { eutróficas) }\end{array}$} \\
\hline & & Idade & Peso & Altura & IMC & Idade & Peso & Altura & IMC \\
\hline \multirow[t]{2}{*}{ Peso } & C. pearson & 0,05 & - & - & - & $-0,27^{*}$ & - & - & - \\
\hline & $p$ & 0,68 & - & - & - & 0,00 & - & - & - \\
\hline \multirow[t]{2}{*}{ Altura } & C. pearson & $-0,05$ & $0,28^{*}$ & - & - & $-0,25^{* *}$ & $0,39^{* *}$ & - & - \\
\hline & $p$ & 0,68 & 0,04 & - & - & 0,00 & 0,00 & - & - \\
\hline \multirow[t]{2}{*}{ IMC } & C. pearson & 0,09 & $0,94^{* *}$ & $-0,03$ & - & $-0,18^{*}$ & $0,90^{* *}$ & $-0,01$ & - \\
\hline & $p$ & 0,49 & 0,00 & 0,81 & - & 0,03 & 0,00 & 0,88 & - \\
\hline \multirow[t]{2}{*}{ Ingestão de cálcio } & C. pearson & $-0,04$ & $-0,69$ & 0,13 & $-0,13$ & $-0,12$ & 0,04 & $0,19^{*}$ & $-0,03$ \\
\hline & $p$ & 0,74 & 0,62 & 0,35 & 0,35 & 0,16 & 0,65 & 0,03 & 0,73 \\
\hline
\end{tabular}

${ }^{*} p<0,05,{ }^{* *} p<0,01$; IMC: índice de massa corporal. 
Ob2= 21 (29,5\% de 71$) ; \mathrm{Eu} 1=3(14,2 \%$ de 21$)$; $\mathrm{Eu} 2=15(27,7 \%$ de 71$) . \mathrm{O}$ mesmo ocorreu no FCA-LD.

Quanto ao Eu1, a perda de uma paciente de $2^{\circ}$ IDR24H e o fato de o grupo ter menos de trinta participantes comprometeram a aplicação do método "EAR como ponto de corte aplicado a grupos". Isso foi observado ao ajustar o consumo de cálcio pela variância intrapessoal e interpessoal, pois o consumo médio que era de $200 \mathrm{mg}$, após o ajuste, passou a ser de $1000 \mathrm{mg}$ de cálcio (Tabela 2). Diante disso, avaliou-se também a ingestão de Ca através do método "EAR como ponto de corte aplicado a indivíduos" nas três pacientes com 2 IDR24H e verificou-se que, em todas elas, a probabilidade da ingestão estar adequada era de $15 \%$. Ou seja, nesse grupo, 14\% das mulheres ( $n=3 / 21)$ apresentaram uma ingestão inadequada de cálcio. Esse resultado corrobora a necessidade de considerar o método aplicado a indivíduos nesse grupo específico (Eu1).

A quantidade de cálcio ingerida (média - M e Mediana - Md), obtida dos recordatórios de 24 horas, antes de aplicar o fator ajuste, foi de $M=245,69$, Desvio-Padrão - $D P=161,27$ e
$M d=241,57$, e de $M=292,63, D P=210,87$ e $M d=242,72$ para o grupo Ob1 e Ob2 respectivamente, e de $M=298,18, D P=187,03$ e $M d=280,54$ e de $M=347,74, D P=361,53$ e $\mathrm{Md}=270,35$ para o grupo Eu1 e Eu2 respectivamente. Após o ajuste, os dados apresentados na Tabela 3 considerando obesas e eutróficas como um todo e os subgrupos Ob1 e Ob2 e Eu1 e Eu2, assim como a ingestão obtida pelo inquérito dietético de frequência de consumo. Os resultados revelaram uma ingestão abaixo do recomendado pelas DRI em ambos os grupos (eutróficas e obesas) e em ambas as faixas etárias (Ob1, Ob2, Eu1 e Eu2), para os dois tipos de inquéritos aplicados.

A Figura 1 ilustra a ingestão de cada um dos indivíduos estudados, obtida segundo IDR24h, comparada à recomendação por faixa etária.

O consumo de fontes alimentares de cálcio em frequência mensal foi relatado por 39,13\% e $17,39 \%$ das mulheres obesas e eutróficas respectivamente, em frequência semanal, por 34,78\% e $26,08 \%$. A frequência diária foi referida por $30,43 \%$ e $17,39 \%$ das mulheres obesas e eutróficas, respectivamente (Figura 2). Em relação ao

Tabela 3. Distribuição dos grupos estudados segundo ingestão de cálcio estimada por inquérito dietético recordatório de $24 \mathrm{~h}$ e de frequência de consumo alimentar de leite e derivados versus recomendações de ingestão diária. Flexeiras (AL), 2007.

\begin{tabular}{|c|c|c|c|c|}
\hline \multirow{2}{*}{$\begin{array}{l}\text { Tipo de ID vs } \\
\text { Grupos e Al }\end{array}$} & \multicolumn{4}{|c|}{ Ingestão de cálcio (mg/dia) } \\
\hline & \multicolumn{2}{|c|}{ Mulheres obesas $(n=102)$} & \multicolumn{2}{|c|}{ Mulheres eutróficas $(n=75)$} \\
\hline \multicolumn{5}{|l|}{ IDR24h* } \\
\hline$O b$ vs Eu & \multicolumn{2}{|c|}{ Total $(\mathrm{Ob} 1=31$ e Ob2=71) } & \multicolumn{2}{|c|}{ Total $(E u 1=21$ e Eu2 $=54)$} \\
\hline Média, DP & \multicolumn{2}{|c|}{$M=281,47, D P=115,32$} & \multicolumn{2}{|c|}{$278,21, \mathrm{DP}=116,00$} \\
\hline Mediana & \multicolumn{2}{|c|}{249,66} & \multicolumn{2}{|c|}{275,28} \\
\hline$I D R 24 h^{*}$ & Ob1: $19-50 a$ & Ob2: $\geq 51 a$ & Eu1: $19-50$ a & Eu2: $\geq 51$ a \\
\hline Ob1 e 2 vs Eu1 e 2 & $(n=31 ; 100 \%)$ & $(n=71 ; 100 \%)$ & $(n=21 ; 100 \%)$ & $(n=54 ; 100 \%)$ \\
\hline Média, DP & $M=261,63, D P=72,86$ & $M=290,13, D P=160,84$ & $M=1000, D P=77,26$ & $M=304,82, D P=139,62$ \\
\hline Mediana & 252,61 & 239,77 & 993,71 & 292,47 \\
\hline \multicolumn{5}{|l|}{ ID FCA-LD } \\
\hline Ob1 e 2 vs Eu1 e 2 & $(n=7 ; 22,58 \%)$ & $(n=16 ; 22,56 \%)$ & $(n=3 ; 14,28 \%)$ & $(n=11 ; 20,3 \%)$ \\
\hline Média, DP & $M=218,25, D P=240,73$ & $M=165,05, D P=224,84$ & $M=43,25, D P=30,73$ & $M=196,96, D P=234,65$ \\
\hline Mediana & 120 & 82,28 & 43,25 & 71,14 \\
\hline $\mathrm{Al}^{* *}$ & 1000 & 1200 & 1000 & 1200 \\
\hline
\end{tabular}




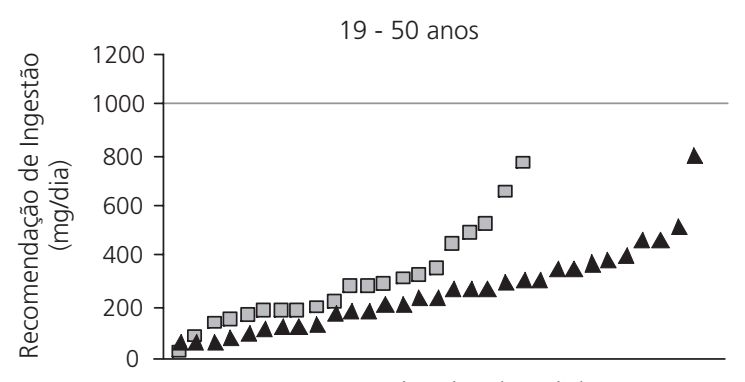

Ingestão de cálcio (mg/dia)

$\square$ Eutróficas $\Delta$ Obesas - Recomendação

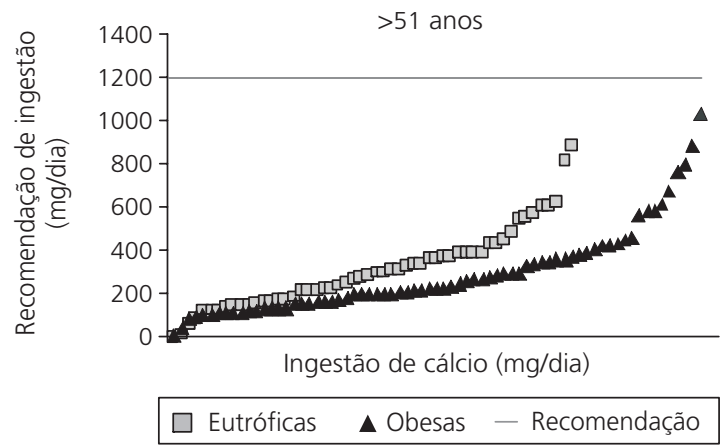

Figura 1. Distribuição dos grupos estudados (triângulo: grupo estudo, mulheres obesas, quadrado: grupo controle, mulheres eutróficas) segundo ingestão de cálcio (mg/dia) por inquérito dietético recordatório de $24 \mathrm{~h}$ versus recomendações de ingestão diária de $1000 \mathrm{mg} / \mathrm{dia}, 19$ a 50 anos e ingestão adequada $=1200 \mathrm{mg} / \mathrm{dia},>51$ anos), sinalizadas na linha horizontal que atravessa o gráfico. Flexeiras (AL), 2007.

número de porções diárias, foi referido o consumo de uma porção/dia por $14,28 \%$ e $4,34 \%$ das eutróficas e obesas, respectivamente e duas porções ou mais por $14,28 \%$ das eutróficas e $21,73 \%$ das obesas. Os dados do FCA-LD revelaram, portanto, um consumo diário muito baixo de fontes alimentares de cálcio de maior biodisponibilidade (provenientes de leite e derivados) em ambos os grupos, destacando-se, também em ambos, a referência a "raramente" consumir leite e derivados (Figura 2).

Os resultados obtidos com os inquéritos dietéticos IDR24H e FCA-LD sinalizam na mesma direção: consumo insuficiente de cálcio.
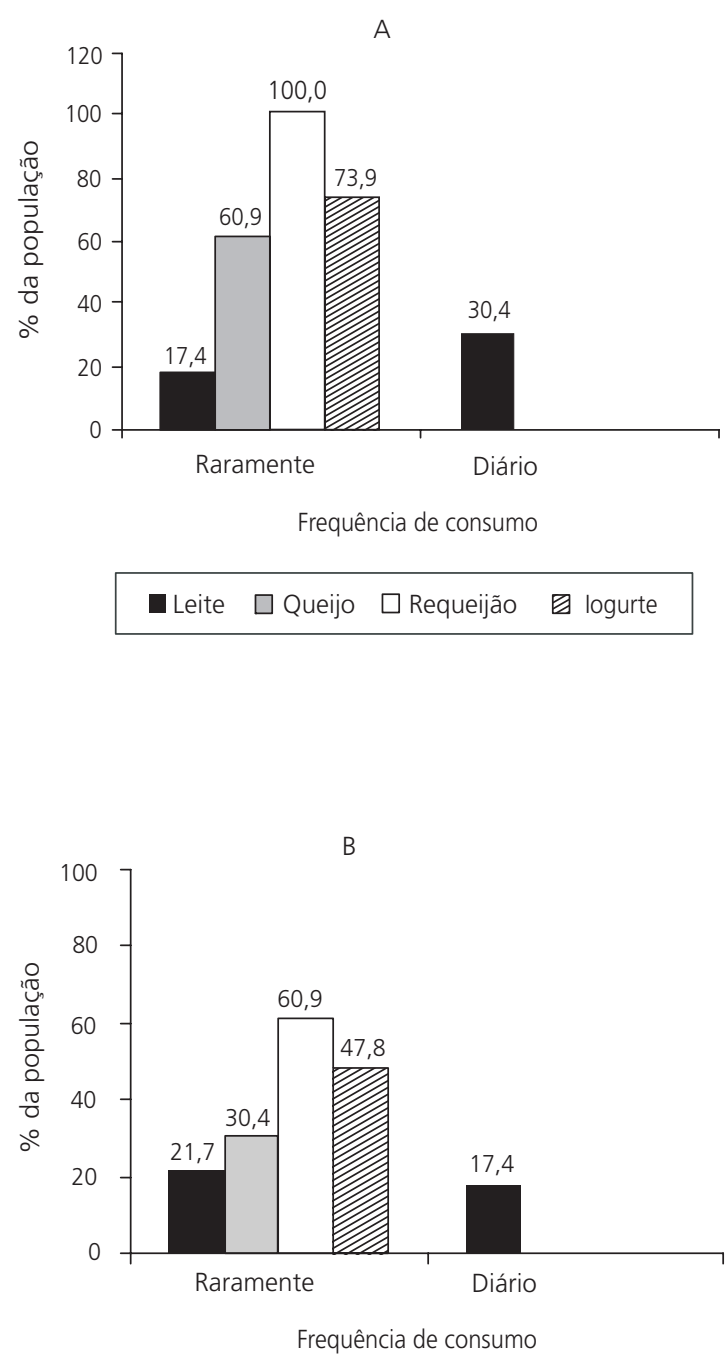

—eite $\square$ Queijo $\square$ Requeijão $\square$ logurte

Figura 2. Frequência de consumo de cálcio proveniente de leite e derivados nos grupos estudados. Flexeiras (AL), 2007.

Nota: A) mulheres obesas e B) mulheres eutróficas.

\section{DISCUSS ÃO}

Neste estudo não houve associação significativa entre ingestão de cálcio e IMC em ambos os grupos. Entretanto, a relação direta observada entre altura e ingestão de cálcio na faixa etária de maiores de 50 anos, considerando a associação direta entre altura e peso, e deste com IMC, permite inferir que haveria uma tendência para associação positiva entre ingestão de cálcio e obesidade. Do ponto de vista teórico, tal associação se 
explica o consumo insuficiente do mineral acarretar maior absorção de gordura e induzir ao aumento de paratormônio (PTH) e de vitamina $D$, que induzem e sustentam a concentração de cálcio intracelular, que, associada à expressão do gene agouti, implica em maior expressão e ativação da ácido graxo sintetase, pelo cálcio, induzindo ao aumento da lipogênese de novo ${ }^{24}$. Como a ingestão observada, no entanto, foi inferior às DRI nos dois grupos, a associação com a obesidade não pode ser confirmada no presente estudo, divergindo do observado em outras investigações ${ }^{11,13,25,26}$, muito embora Heaney ${ }^{27}$, observando a ingestão de cálcio e densidade óssea em mulheres, tenha constatado que o grupo com ingestão no percentil 25 apresentava 15\% de prevalência de sobrepeso; em contrapartida, o grupo com ingestão de acordo com a recomendação teve apenas $4 \%$ de sobrepeso. Dentre aqueles estudos que encontraram relação inversa entre consumo de Ca e obesidade, destacam-se dois recentes: um avaliou dois grupos de obesos, submetidos à restrição energética, com ou sem suplementação de cálcio dietético durante quatro meses, e observaram que o grupo em dieta rica em cálcio apresentou redução significativa da circunferência da cintura, sugerindo que a dieta rica em cálcio pode potencializar os efeitos da restrição energética sobre a obesidade abdominal28; o outro avaliou mulheres segundo faixas de IMC e observou após 30 meses que as obesas consumiam significativamente menos cálcio e que as eutróficas consumiam mais leite ${ }^{29}$.

De forma semelhante ao observado neste estudo, porém, Snijder et al..$^{30}$ não encontraram associação; eles avaliaram 2064 indivíduos entre 50 e 75 anos, e verificaram que o consumo elevado de cálcio não apresentou associação com menor peso corporal ou melhores níveis dos componentes da síndrome metabólica, exceto com pressão arterial. Resultados também semelhantes foram verificados por Oliveira et al. ${ }^{31}$, com 85 mulheres no climatério: a ingestão de cálcio não apresentou correlação significante com o IMC.

Ao comparar a frequência de consumo de fontes de cálcio do presente estudo à recomendada pelo guia alimentar para a população brasileira ${ }^{32}$, verificou-se que, entre as consumidoras de porções diárias de tais fontes alimentares, todas, de ambos os grupos, independentemente da faixa etária, apresentavam consumo abaixo do recomendado: três porções diárias, correspondentes à faixa de $800 \mathrm{mg}$ a $1200 \mathrm{mg}$ de cálcio ${ }^{33}$. Considerando as características da população estudada (idade, obesidade, DM e HAS), deve-se estimular o consumo de leite e derivados por serem fontes de maior biodisponibilidade do mineral e pela ação do cálcio na regulação da hipertensão, na resistência à insulina e na obesidade, pois desequilíbrios do fluxo de cálcio extracelular, ou de sua sinalização, podem representar fatores fundamentais para estabelecer uma relação de causalidade entre essas três condições ${ }^{25}$.

Vale salientar que a avaliação da FCA-LD enfoca apenas as fontes de maior biodisponibilidade, deixando-se de considerar o cálcio obtido do consumo de outros alimentos, como os vegetais, muitas vezes as maiores fontes na dieta da população, principalmente em população de baixa renda como a aqui estudada, cuja renda per capita é de $R \$ 64,34$ (http://www.frigoletto.com.br/ GeoAlagoas/idhal.htm) ${ }^{34}$. Esse aspecto é corroborado pela análise do consumo por meio do IDR24h, que foi muito superior ao encontrado pela FCA-LD. Ficou claro, mediante os dois tipos de inquéritos utilizados, que o consumo de cálcio é baixo nessa população.

Em função de o grupo estudado ser composto por mulheres, o baixo consumo de cálcio torna-se preocupante por contribuir para futuros problemas de osteoporose. Apesar de o esqueleto funcionar como uma reserva grande de cálcio para a manutenção das concentrações no líquido extracelular e funções celulares, a deficiência no nível celular e tecidual essencialmente nunca é encontrada. Seja qual for a redução dessa reserva, ela implicará a diminuição da resistência óssea, comprometendo a função mecânica do esqueleto. Além disso, os adultos perdem massa óssea a uma taxa de aproximadamente $1 \%$ ao ano, e isso culmina com as alterações que ocorrem na menopausa: ingestão declinante de cálcio, redução da 
atividade física, diminuição dos hormônios sexuais, da concentração de vitamina $D$ ativa e da resistência intestinal ${ }^{35}$. Outro aspecto igualmente importante é a relação entre osteoporose, síndrome metabólica e obesidade andróide, cuja plausibilidade biológica apresentada em revisão atual ${ }^{10}$ foi discutida e deve ser considerada.

O baixo consumo de cálcio é resultado da própria evolução humana, pois o homem primitivo consumia em média $1500 \mathrm{~g}$ de cálcio/dia de raízes, tubérculos, nozes e feijões. Com a utilização de cereais, a ingestão de cálcio diminuiu substancialmente, porque os alimentos básicos tornaram-se os grãos (frutos), parte do vegetal que possui menos cálcio. O homem moderno consome, em média, cálcio insuficiente para otimizar a densidade óssea ${ }^{36}$. Os principais motivos para a baixa ingestão de cálcio observada na população brasileira são decorrentes do hábito alimentar, com substituição do leite por bebidas com baixo teor de cálcio, como os refrigerantes, refeições fora de casa, além do alto custo dos alimentos com maior biodisponibilidade ${ }^{37}$. Na dieta ocidental, as maiores fontes de cálcio são leite e derivados. Como a população estudada consome pouco esses alimentos, é provável que o cálcio dietético seja obtido predominantemente de vegetais. Para esse perfil de ingestão, cabe ressaltar ainda que, mesmo obtendo na dieta quantidades suficientes de cálcio a partir dos vegetais, outros componentes desses alimentos podem diminuir sua biodisponibilidade, como ácido oxálico, ácido fítico, fibras etc. $^{35}$, e pode, consequentemente, comprometer sobremaneira a utilização do cálcio.

\section{ONCLUS Ã O}

O presente estudo não verificou associação positiva significativa, podendo apenas inferir uma tendência ao consumo deficiente de cálcio estar associado à obesidade. No entanto, o estudo revelou um achado relevante: o consumo quantitativo (IDR24H) - obtido do cálcio total ingerido de vegetais, leite e derivados - e o qualitativo (FCA-LD) - obtido apenas de fontes de maior biodisponibilidade do mineral - foram insuficientes para atingir as quantidades recomendadas tanto entre as mulheres obesas quanto entre as eutróficas estudadas. Considerando tratar-se de um grupo acompanhado pelo programa de controle da HAS e diabete mellitus, é evidente a necessidade de atividades de educação nutricional para estimular o consumo de fontes alimentares de cálcio de maior biodisponibilidade como parte de uma dieta adequada. Além disso, o mineral é importante auxiliar no controle da HAS e na prevenção de agravos importantes, como a osteoporose, assim como sua falta é fator de risco em potencial para a obesidade.

Nesse sentido, os resultados obtidos neste estudo foram disponibilizados para as equipes da estratégia de saúde da família que assistem tais pacientes, bem como para a população estudada.

\section{A GRADECIMENTOS}

Às pacientes e à Secretaria Municipal de Saúde de Flexeiras (AL). Às instituições de financiamento MS-DECIT-PPSUS/CNPq/FAPEAL/SESAU-AL e à revisora Ana Maria Vasconcelos Martins de Castro.

\section{COLABORADORES}

P.M.C. SILVA participou da definição do trabalho, coleta, tabulação, análise dos dados e escrita do artigo. C.R. CABRAL JUNIOR participou da análise dos dados e escrita do trabalho. S.M.L. VASCONCELOS participou da definição do trabalho, coleta, análise dos dados e escrita do artigo. Coordenadora do projeto de pesquisa para o SUS e orientadora do trabalho.

\section{REFERÊ NCIAS}

1. Carvalho KMB. Obesidade. In: Cuppari L. Guia de nutrição: nutrição clínica no adulto. $2^{a}$ ed. Barueri: Manole; 2005.

2. Choban PS, Jackson B, Poplawski S, Bistolarides P. Bariatric surgery for morbid obesity: why, when, how, where and then what? Clev Clin J Med. 2002; 69(11):897-903. doi: 10.3949/ccjm.69.11.897. 
3. Detel M, Shikora SA. The development of the surgical treatment of morbid obesity. J Am Coll Nutr. 2002; 21(5):365-71.

4. MacDonald Jr. KG. Overview of the epidemiology of obesity and the early history of procedures to remedy morbid obesity. Arch Surg. 2003;138(4): 357-60.

5. Marques-Lopes I, Marti A, Moreno-Aliaga MJ, Martinez A. Aspectos genéticos da obesidade. Rev Nutr. 2004;17(3):327-38. doi: 10.1590/S1415-52 732004000300006.

6. Popkin BM. The nutrition transition in low-income countries: an imerging crisis. Nutr Rev. 1994; 52(9): 285-98.

7. Monteiro CA, Mondini L, Costa RBL. Mudanças na composição e adequação nutricional da dieta familiar nas áreas metropolitanas do Brasil (1988-1996). Rev Saúde Pública. 2000; 34(3):251-8.

8. Garcia RWD. Reflexos da globalização na cultura alimentar: considerações sobre as mudanças na alimentação urbana. Rev Nutr. 2003;16(4):483-92. doi: 10.1590/S1415-52732003000400011.

9. Cozzolino SMF. Minerais e obesidade. In: Cozzolino SMF. Biodisponibilidade de nutrientes. São Paulo: Manole; 2005. p.878.

10. Rosen CJ, Klibanski A. Bone, fat, and body composition: evolving concepts in the pathogenesis of osteoporosis. Am J Med. 2009; 122:409-14. doi:10.1016/j.amjmed.2008.11.027.

11. Schrager S. Dietary calcium intake and obesity. J Am Board Fam Pract. 2005; 18(3):205-10.

12. McCarron DA. Calcium and magnesium nutrition in human hypertension. Ann Intern Med. 1983; 98(5):800-5.

13. Zemel MB, Shi H, Greer B, Dirienzo D, Zemel PC. Regulation of adiposity by dietary calcium. FASEB J. 2000; 14(9):1132-38.

14. Dubnov-Raz G, Berry EM. The dietary treatment of obesity. Endocrinol Metab Clin N Am. 2008; 37: 873-86.

15. Major GC, Chaput JP, Ledoux M, St-Pierre S, Anderson $\mathrm{GH}$, Zemel MB, et al. Recent developments in calcium-related obesity research. Obes Rev. 2008; 9:428-45. doi: 10.1111/j.1467-789X.2007. 00465.x.

16. Kamycheva E, Joakimsen RM, Jorde R. Intakes of calcium and vitamina $D$ predict body mass index in the population of northern Norway. J Nutr. 2003; 133(1):102-6.

17. Astrup A. The role of calcium in energy balance and obesity: the search for mechanisms. Am J Clin Nutr. 2008; 88:873-74.
18. Santos LC, Martini LA, Freitas SN, Cintra IP. Ingestão de cálcio e indicadores antropométricos entre adolescentes. Rev Nutr. 2007; 20(3):275-83. doi: 10.1590/S1415-52732007000300006.

19. Zabotto CB, Vianna RPT, Gil MF. Registro fotográfico para inquéritos dietéticos, utensílios e porções. Goiânia: Metha; 1996.

20. Philippi, ST. Virtual nutri. versão 1.0. [software] for windows. São Paulo: Universidade de São Paulo; 1996.

21. Brasil. Ministério da Saúde. Vigilância Alimentar e Nutricional. SISVAN: orientações básicas para a coleta, o processamento, a análise de dados e a informação em serviços de saúde. Brasília: Ministério da Saúde; 2004.

22. Fisberg RM, Slater B, Marchioni DML, Martini LA. Inquéritos alimentares: métodos e bases científicos. Barueri: Manole; 2005.

23. National Research Council. Dietary reference intakes for calcium, phosphorus, magnesium, vitamin D and fluoride. Washington (DC): National Academy Press; 1999.

24. Yanovski JA, Parikh SJ. Calcium intake and adiposity. Am J Clin Nutr. 2003; 77(2):281-87.

25.Zemel MB, Kim JH, Woychik RP, Michaud EJ, Kadwell SH, Patel IR, et al. Agouti intracellular calcium: role in the insulin resistance of viable yellow mice. Proc Natl Acad Sci. 1995; 92(11): 4733-7.

26. Zemel MB. Regulation of adiposity and obesity risk by dietary calcium: mechanisms and implications. J Am Coll Nutr. 2002; 21(2):146-51.

27. Heaney RP. Normalizing calcium intake: projected population effects for body weight. J Nutr. 2003; 133(1):268-70.

28. Torres MSG, Abreu VG, Francischetti EA, Nogueira LP, Todesco L, Miceli D, et al. Efeitos do cálcio dietético sobre a perda ponderal, obesidade abdominal, perfil metabólico e níveis de pressão arterial em obesos submetidos à restrição calórica. Resumos do $14^{\circ}$ Hipertensão. Congresso Brasileiro de Hipertensão; 2008.

29. Dicker D, Belnic Y, Goldsmith R, Kaluski DN. Relationship between dietary calcium intake, body mass index, and waist circumference in MABAT: the Israeli National Health and Nutrition Study. IMAJ. 2008; 10:512-15.

30. Snijder MB, van der Heijden AAWA, Dam RM, Stehouwer CDA, Hiddink GJ, Nijpels G, et al. Is higher dairy consumption associated with lower body weight and fewer metabolic disturbances? The Hoorn Study. Am J Clin Nutr. 2007; 85: 989-95. 
31. Oliveira JR, Assumpção SR, Sotelo YM, Bon AX. Consumo de cálcio e relação cálcio/proteína da dieta em mulheres no climatério. Nutrire: Rev Soc Bras Aliment Nutr. 2007; 32(S):233.

32. Brasil. Ministério da Saúde. Guia alimentar para a população brasileira: promovendo a alimentação saudável. Brasília: Ministério da Saúde; 2006.

33. Philippi ST, Laterza AR, Cruz ATR, Ribeiro LC. Pirâmide alimentar adaptada: guia para a escolha dos alimentos. Rev Nutr. 1999; 12(1):65-80. doi: 10.1590/S1415-52731999000100006.

34. Menezes EF. Frigoletto.com.br: a geografia em primeiro lugar. [Internet]. Maceió; 2000 [acesso 2009 out 26]. Disponível em: <http://www. frigoletto.com.br/GeoAlagoas/idhal.htm>.
35. Weaver CM, Heaney RP. Cálcio. In: Shils ME, Olson JA, Shike M, Ross AC. Tratado de nutrição moderna na saúde e na doença. $9^{a}$ ed. São Paulo: Manole; 2003. v. 1

36. Eaton SB, Konner M. Paleolithic nutrition: a consideration of its nature and current implications. N Engl J Med. 1985; 312(5):283-9.

37. Pereira GAP, Genaro PS, Pinheiro MM, Szejnfeld VL, Martini LA. Cálcio dietético: estratégias para otimizar o consumo. Rev Bras Reumatol. 2009; 49(2):164-80.

Recebido em: 18/4/2008

Versão final reapresentada em: 12/11/2009

Aprovado em: 17/3/2010 